\title{
HYMENOMYCETES DETERIORADORES DE MADEIRA EM SERRARIAS DE MANAUS, AM, BRASIL. ${ }^{1}$
}

\author{
Maria N. S. RIBEIRO ${ }^{2,}$ Izonete de J. A. AGUIAR ${ }^{2}$
}

\begin{abstract}
RESUMO - Do levantamento realizado com fungos (Hymenomycetes) deterioradores de madeiras em serrarias de Manaus foram encontradas as seguintes espécies: Coriolopsis occidentalis, Pleurotus ostreatus, Pycnoporus sanguineus e Schizophyllum commune. Pleurotus ostreatus foi assinalada com maior freqüência. As seis espécies de madeiras examinadas Ceiba pentandra (sumaúma), Copaifera multijuga (copaiba), Hura crepitans (assacu), Maquira coriacea (muiratinga), Pseudobombax mumguba (munguba) e Virola surinamensis (virola) tiveram suas cascas e alburnos atacados. Hura crepitans foi a que apresentou maior incidência de fungos.
\end{abstract}

Palavras-chave - Hymenomycetes apodrecedores; Madeiras amazônicas; Serrarias.

Deomposing Hymenomycetes of Wood in Sawmills of Manaus, AM, Brazil.

ABSTRACT - A survey of wood decomposing (Hymenomycetes) fungi in sawmills of Manaus, Amazonas, revealed the following species: Coriolopsis occidentalis, Pleurotus ostreatus, Pycnoporus sanguineus and Schizophyllum commune. Pleurotus ostreatus was the most frequent. The six commercial tree species examined were Ceiba pentandra (sumaúma), Copaifera multijuga (copaiba), Hura crepitans (assacu), Maquira coriacea (muiratinga), Pseudobombax munguba (munguba) and Virola surinamensis (virola). All showed signs of infestation in the bark and sapwood, Hura crepitans showed the highest incidence of fungal reproductive parts.

Key words - Decomposing Hymenomycetes, Amazon wood, Sawmills.

\section{INTRODUÇÃO}

Os fungos são organismos encontrados nos mais variados substratos, entre os quais se destaca a madeira, interferindo em suas propriedades físicas, químicas e mecânicas, causando sérios danos econômicos ao setor florestal e madeireiro.

Entre os fungos que causam prejuizo à madeira, destacam-se os Basidiomycotina, onde são encontradas a maioria das espécies xilófagas. Estas espécies pertencem à classe dos Hymenomycetes ou Basidiomycetes propriamente dita. Segundo PINTO-
LOPES(1950), algumas espécies poliporóides atacam madeira provocando podridão e que, em árvores vivas, geralmente acarretam-lhes a morte. GILBERTSON (1980) menciona que a maioria das espécies xilófagas pertence à ordem Aphyllophorales destacando a familia Polyporaceae.

Os levantamentos de fungos xilófagos no Brasil são escassos, especialmente em serrarias, ressalvando-se os trabalhos realizados por CAVALCANTI (1983) nas serrarias de Recife, Pernambuco e por COÊLHO (1991) nas serrarias de Manaus,Amazonas.

\footnotetext{
1 Resumo da Monografía apresentada para obtenção do grau de Engenheiro Florestal pelo Instituto de Tecnologia da Amazônia-UTAM, Manaus-AM.

2 Instituto Nacional de Pesquisas da Amazonia -INPA.
} 
O presente trabalho foi desenvolvido visando caracterizar os fungos Hymenomycetes que atacam as madeiras estocadas em serrarias de Manaus, considerando a incontestável importância dos fungos xilófagos, principalmente quando tomando como parâmetro o prejuízo financeiro que certas espécies podem causar e a falta de conhecimento básico sobre esses fungos na região.

\section{MATERIAL E MÉTODOS}

No período de novembro de 1991 a maio de 1992, coletou-se mensalmente fungos Hymenomycetes em seis espécies de madeira, em quatro serrarias de Manaus-AM.

As informações relativas aos nomes vulgares, procedência e utilização das madeiras foram obtidas nos locais de coleta.

Após cada coleta, foi feita a descrição macroscópica dos fungos ainda frescos, seguindo-se a secagem em estufa a $60^{\circ} \mathrm{C}$. Para identificação da coloração dos basidiocarpos, usou-se a carta de cores de LOCQUIN (1975), abreviada para $\mathrm{L}$.

$\mathrm{Na}$ análise das microestruturas obedeceu-se as técnicas propostas por MARTIN (1934) e TEIXEIRA (1956) e na das reações de amiloidia e cianofilia as de SINGER (1975) e KOTLABA \& POUZAR (1964), respectivamente.

A identificação dos fungos foi baseada principalmente em DENNIS (1970) e CAVALCANTI (1976). A das madeiras foi feita por especialistas do Setor de Anatomia da Madeira, do INPA. A terminologia micológica segue FIDALGO \& FIDALGO (1967).

Os espécimes de fungos encontram-se depositados no Herbário do Instituto Nacional de Pesquisas da Amazônia (INPA).

\section{RESULTADOS E DISCUSSÃo}

Nas toras de madeira (Tab. I) depositadas nas quatro serrarias onde foram procedidas as coletas, detectouse quatro espécies de fungos Hymenomycetes: Coriolopsis occidentalis (Klotz.) Cunn., Pleurotus ostreatus Jacq. Pycnoporus sanguineus (L.:F.) Murr. (Polyporaceae) e Schizophyllum commune Fr. (Schizophyllaceae).

Tabela 1. Madeiras atacadas por fungos Hymenomyetes.

\begin{tabular}{|c|c|c|c|}
\hline Noma Clentiflco & Nome Vulgar & Habltat & Utllização \\
\hline $\begin{array}{l}\text { Ceiba pentandrs } \\
\text { Gaertin(Bombacsceas }\end{array}$ & Sumaùma & $\begin{array}{l}\text { Mata de } \\
\text { várzea e } \\
\text { Terra firme }\end{array}$ & $C, D, E$ \\
\hline $\begin{array}{l}\text { Copaifera multijuga } \\
\text { Hayne(Caesalpiniacea }\end{array}$ & Copalba & $\begin{array}{l}\text { Mata de terra } \\
\text { firme }\end{array}$ & B \\
\hline $\begin{array}{l}\text { Hura crepitans L. } \\
\text { (Euphortiaceae) }\end{array}$ & Assecu & $\begin{array}{l}\text { Mata de } \\
\text { várzea }\end{array}$ & $A, D$ \\
\hline $\begin{array}{l}\text { Mequira coriacea CC. } \\
\text { Berg. (Moraceae) }\end{array}$ & Muratinga & $\begin{array}{l}\text { Mata de } \\
\text { várzeo }\end{array}$ & C. D \\
\hline $\begin{array}{l}\text { Pseudobombax } \\
\text { munguba (Mart. et } \\
\text { Zucc. (Bombaceae) }\end{array}$ & Mungubs & $\begin{array}{l}\text { Mata de } \\
\text { varzea }\end{array}$ & $E$ \\
\hline $\begin{array}{l}\text { Virola surinamensis } \\
\text { (Rol.) Wart } \\
\text { (Myristicaceae) }\end{array}$ & Virola & $\begin{array}{l}\text { Mata de } \\
\text { várzea }\end{array}$ & $A, B, D, E$ \\
\hline
\end{tabular}

LEGENDA: A) compensados; B) marcensria; C) brinquedos: D) caixas e E) fabricaçăo de papel.

Os representantes de Pleurotus ostreatus ocorreram em todas as toras de madeira estudadas, Pycnoporus sanguineus ocorreu nas madeiras 
Tabela 2. Ocorrência de Hymenomycetes em toras de madeira em serrarias de Manaus.

\begin{tabular}{lcccccc}
\hline \multirow{2}{*}{ Fungo } & \multicolumn{7}{c}{ Hospedeiro } \\
\cline { 2 - 7 } & Assacu & Virola & Sumaúma & Muiratinga & Copaiba & Munguba \\
\hline C. occidentalis & + & + & - & + & - & - \\
P.ostreatus & + & + & + & + & + & + \\
P. sanguineus & + & - & - & + & + & - \\
S.commune & + & - & - & + & - & - \\
\hline
\end{tabular}

Legenda: $(+)$ - presença; $(-)$ - ausència.

assacu, copaiba e muiratinga. Coriolopsis occidentalis foi encontrado nas toras de assacu e virola e, Schizophyllum commune apenas em assacu (Tab. 2). Com relação as madeiras estudadas, o percentual de freqüência dos fungos corresponde respectivamente a $100 \%, 50 \%, 33 \%$ e $16.6 \%$.

As espécies estudadas colonizaram a casca e o alburno das toras, com exceção de Pleurotus ostreatus, as demais causaram podridão branca. DIROL \& FOURGEROUSSE (1979) citam Schizophyllum commune, OFOSU-ASIEDU (1979) e GILBERTSON \& RYVARDEN (1987) mencionam Coriolopsis occidentalis $\mathrm{e}$ Pycnoporus sanguineus, como causadores de podridão branca em dicotiledôneas, o que confirma os resultados obtidos no presente trabalho.
De acordo com a literatura consultada não existe referências para o tipo de podridão causada por Pleurotus ostreatus. Posteriormente, poderão ser desenvolvidas pesquisas que possibilitem a identificação do tipo de podridão causada por Pleurotus ostreatus.

Os fungos acima com exceção de Pleurotus ostreatus também foram encontradospor JESUS \& BONONI (1991), atacando as madeiras de melancieira (Alexa grandiflora), pau marfim (Aspidosperma obscurinervium), pau rainha (Brosimum rubescens), tanimbuca (Buchenavia oxycarpa), louro (Licaria aritu), mata-mata (Eschweilera sp.), ucuubarana (Osteophloeum platyspermum) e virola (Virola sp.), estocadas "in situ" na área da Usina Hidrelétrica de Balbina, no municipio de Presidente Figueiredo (Amazonas), o que demonstra a não especificidade destes

\section{Chave para identificação dos Hymenomycetes estudados}

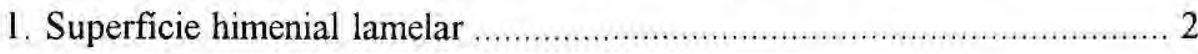

1. Superficie himenial poróide ............................................................ 3

2. Estipe presente, tomentoso ...........................Pleurotus ostreatus

2. Estiper ausente ....................................... Schizophyllum commune

3. Basidiocarpo castanho; superficie abhimenial concentricamente zonada; poros 1-2 por mm Coriolopsis occidentalis

3. Basidiocarpo vermelho; superficie abhimenial não concentricamente zonada; poros 4-5 por $\mathrm{mm}$ Pycnoporus sanguineus 
fungos quanto a espécie de madeira.

Segundo BONONI (1984), Schizophyllum commune é muito comum e frequente onde haja madeira em decomposição e que não existia na literatura brasileira a descrição desta espécie, sendo a do presente trabalho a primeira para o Brasil.

\section{Caracteres das espécies}

Coriolopsis occidentalis (Klotz.) Cunn. J. Bot. 19:230.1972. (Fig.1)

Basidiocarpo anual, séssil a efuso reflexo; píleo dimidiado a flabeliforme, 7.5 - $9.5 \times 5.5-6.5 \times 0.6 \mathrm{~cm}$; superfi- cie abhimenial concentricamente zonada e sulcada, castanho amarronzada (LJ2d); margem fina, ondulada a lobada, amarronzada (LA3f); contexto heterogêneo, castanho-claro (LE5h); $0.2-0.6 \mathrm{~cm}$ espesso; superficie himenial poróide, castanha (LJ2d); poros irregulares a angulares, $1-2$ por $\mathrm{mm}$.

Sistema hifálico trimítico; hifa generativa de parede fina, septada, ramificada, 2.0 - 3.5 ìm diâm.; hifa esquelética de parede espessa, asseptada, sem ramificação $3.0-6.5 \mathrm{im}$ diâm.; hifa conjuntiva de parede espessa, asseptada ramificada, $1.5-2.5 \mathrm{im}$
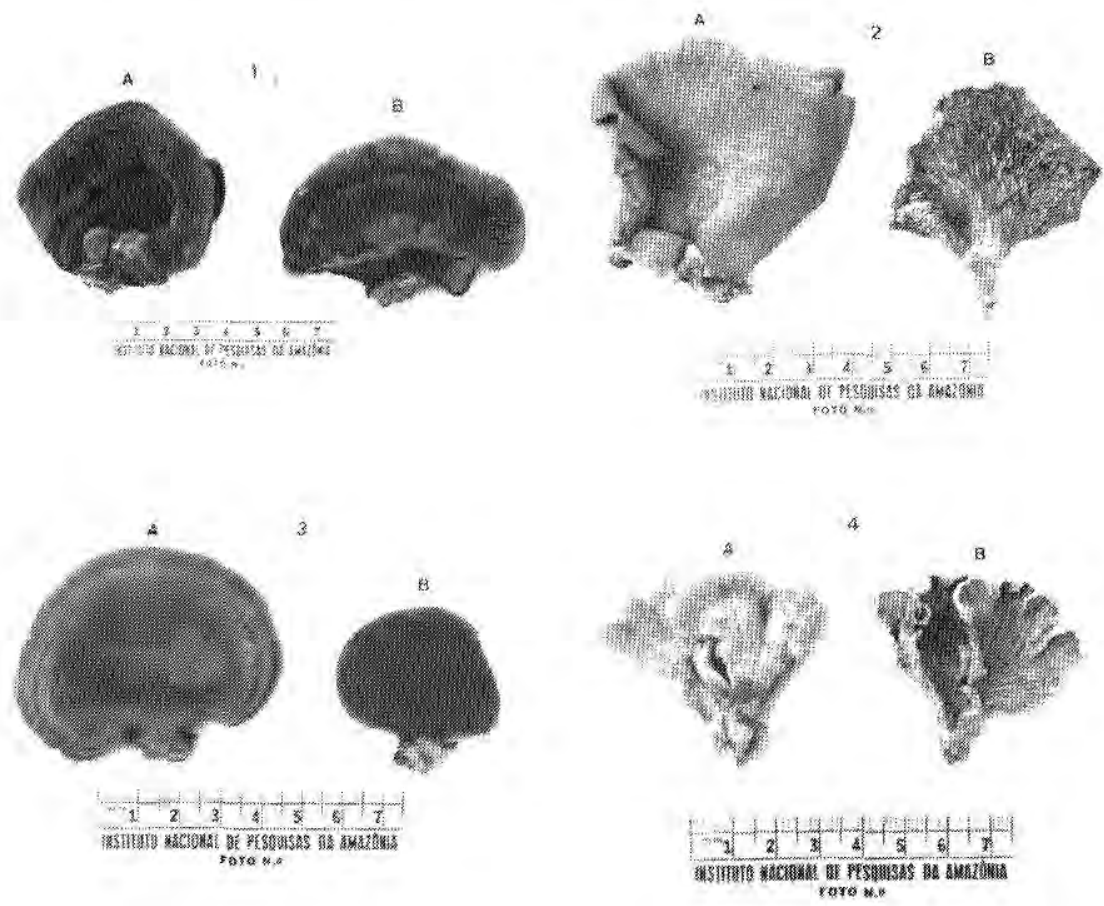

Figuras 1-4. Basidiocarpos. 1-Coriolopsis occidentalis, 2 - Pleurotus ostreatus, 3 Pycnoporus sanguineus, 4 -Schizophyllum commune. (A) Superficie abhimenial, (B) Superficie himenial 
diâm.; himênio com cistídio ausente e medas cônicas $20-70 \times 15-25 \mathrm{im}$; basídio clavado, tetraesterigmado, 9.5 - 13 x 3.0 - $4.0 \mathrm{im}$; basidiosporo cilíndrico, inamilóide e acianófilo, 3.5 $-7.0 \times 2.5-3.5 \mathrm{im}$.

Espécime examinado: Sobre tora de assacu, col. M. N. S. Ribeiro \& S. M. Veras, 4-XI-1991 (INPA 161.680).

Distribuição geográfica: Ocorre nas regiões tropicais do continente americano. É referida para o Amazonas por JESUS \& BONONI (1991).

Pleurotus ostreatus Jacq. Syll. Fung, 5:355.1887 (Fig. 2)

Basidiocarpo anual, estipitado; píleo plano a infudibuliforme, 3.0 - 9.0 $\mathrm{X} 4.5-8.0 \times 0.2-0.3 \mathrm{~cm}$; estipe curto, marrom claro (LM6h), 1.0 - 2.5 $\mathrm{X} 0.2-0.4 \mathrm{~cm}$; superficie abhimenial velutina a pubescente, margem plana quando fresca, involuta quando seca, concolor com a superficie abhimenial; contexto homogêneo, creme-claro (LX8h), 0,7 cm espesso; superfície himenial lamelar creme-amarronzada (LN6h).

Sistema hifálico dimítico; hifa generativa, de parede fina $3.5-4.5 \mathrm{im}$ diâm.; hifa esquelética de parede e diâmetro bem espesso, asseptada, sem ramificação 4.5 - 6.0ìm diâm.; himênio com cistídio metulóide 25 - $39 \times 7,0$ $9,5 \mathrm{im}$; basídio colapsado; basidiosporo hialino, elipsóide, inamilóide e acianófilo, $6,0-10 \times 4,0-5,0 \mathrm{im}$ com um pequeno apículo.

Espécime examinado: Sobre tora de virola, col. M. N. S. Ribeiro \&
S. M. Veras, 4-II-1992 (INPA 161.682).

Distribuição geográfica: Cosmopolita. É registrada para Pernambuco por BATISTA \& BEZERRA (1960).

Pycnoporus sanguineus (L.:Fr.) Murr. Bull. Torrey Bot. Club 31:421.1904. (Fig. 3)

Basidiocarpo anual, séssil a subestipitado; píleo dimidiado a flabeliforme, coriáceo, 4.5-7.0 $\times 3.5-4.5 \times 0.1-0.2$ $\mathrm{cm}$; superficie abhimenial levemente tomentosa, vermelho-sanguinea (LAlh); margem inteira, fina, ondulada, concolor com a superficie abhimenial; contexto homogêneo, creme-avermelhado (LA4h), 0.02-0.1 cm espesso; superficie himenial poróide, laranja-avermelhada (LAlg), com poros pequenos, regulares a angulares, $4-5$ por $\mathrm{mm}$.

Sistema hifálico trimítico; hifa generativa de parede fina, septada, ramificada 2.0 - 3.0 im diâm.; hifa esquelética de parede espessa, asseptada, sem ramificação, 5.0 -7.0 ìm diâm.; hifa conjuntiva de parede espessa, asseptada, ramificada, 1.5 - 2.4 ìm diâm; himênio com cistidio e meda ausente; basídio clavado, tetraesterignado $8-10 \times 2.5$ $5.0 \mathrm{im}$; basidiosporo cilíndrico, inamilóide e acianófilo, 2.5 - $5.0 \times 1.5$. $3.0 \mathrm{im}$.

Espécime examinado: Sobre tora de assacu, col. M. N. S. Ribeiro \& R. J. Ribeiro, 4-I-1992 (INPA 161.681).

Distribuição geográfica: Regiões tropical e subtropical dos hemisférios Norte e Sul. É citada para o Amazonas por JESUS \& BONONI (1991) e COELHO (1991). 
Schizophyllum commune Fr. Systema Mycologicum 1:330.1831. (Fig. 4)

Basidiocarpo anual; píleo séssil a curto-estipitado, flabeliforme, 5.5 - 7.0 $\times 4.0-5.0 \times 0.1 \mathrm{~cm}$; superficie abhimenial, vilosa, pálida a marrom acinzentada (LF5g); margem lobada, involuta, concolor com a superficie abhimenial; contexto homogêneo $0.1 \mathrm{~cm}$ espesso, mais claro que a superficie himenial (LQ7h); superfície himenial lamelar, ligeiramente mais escura que a superficie abhimenial.

Sistema hifálico monomitico; hifa generativa de parede delgada a espessada, hialina, ambas com grampo de conexão, 2.0 - 4.5 ìm diâm.; himênio com cistídio ausente; basídio clavado, tetraesterigmado, $15-20 \times 4.0-5.5$ im; basidiosporo cilíndrico, inamilóide e acianófilo, $3.0-6.0 \times 2.0-3.5 \mathrm{im}$.

Espécime examinado: Sobre tora de assacu, Col. M. N. S. Ribeiro \& S. M. Veras, 04-IV-1992 (INPA 161.683).

Distribuição geográfica: Cosmopolita. É mencionada para o Amazonas, por JESUS \& BONONI (1991).

\section{CONCLUSÕES E SUGESTÕES}

O presente trabalho permitiu as seguintes conclusões:

- Os fungos xilófagos encontrados são da classe dos Hymenomycetes, pertencente as familias Polyporaceae e Schizophyllaceae;

- Coriolopsis occidentalis, Pleurotus ostreatus, Pycnoporus sanguineus e Schizophyllum commune foram encontrados nas toras de assacu (Hura crepitans), sumaúma (Ceiba pentandra), copaiba (Copaifera multijuga), muiratinga (Maquira coriacea), munguba (Pseudobombax munguba) e virola (Virola surinamensis);

- Pleurotus ostreatus foi a espécie mais comum;

- Os fungos encontrados atacaram a casca e o albumo das seis espécies de madeira estudadas; e

- As toras de assacu, foram as que apresentaram maior incidência de fungos.

As condições de estocagem das toras de madeiras nas serrarias, juntamente com a elevada temperatura e umidade local, favoreceram o desenvolvimento destes fungos xilófagos. Estes, ao decomporem a madeira, podem ocasionar grandes prejuizos econômicos à empresa. Entretanto, tais danos podem ser perfeitamente minimizados, quando são adotadas medidas profiláticas simples, como: transporte rápido entre o local de abate e as serrarias; beneficiamento imediato das toras; limpeza do pátio de estocagem; irrigação constante da parte emersa da tora; rolagem constante das toras mantidas no rio; e estocagem das toras sem o contato direto com o solo.

\section{AGRADECIMENTOS}

As autoras agradecem ao $\mathrm{Sr}$. Ralfh João Ribeiro (INPA) pela digitação do texto, a Prof "Solange de Mello Veras (UTAM) pela colaboração nas coletas, a Dra. Maria Auxiliadora 
Cavalcanti (UFPE) pelas sugestões e aos pesquisadores do Setor de Anatomia de Madeira da Coordenação de Pesquisas em Produtos Florestais do INPA, pela identificação das madeiras.

\section{Bibliografia citada}

BATISTA, A. C.; BEZERRA, J. L. 1960. Basidiomycetes vulgares em o nordeste brasileiro. Instituto de Micologia, 294:29.

BONONI, V. L. 1984. Basidiomicetos do Cerrado da Reserva Biológica de MogiGuaçu, S.P. Rickia, 11:1-26.

CAVALCANTI, M. A. Q. 1976. Introdução ao conhecimento dos hasidiomicetos poliporóides da zona da mata de Pernambuco. Tese de Livre Docência, Univ. Fed. Pernambuco - UFPE, Recife, $200 \mathrm{p}$.

1983. Basidiomicetos poliporóides destruidores de madeiras em serrarias do Recife. Rev. Penuamb. Tecnol, 3(3):8387.

COELHO, R. J. F 1991. Levantamento da ocorrência de podridão por Pycnoporus sanguineus em madeiras amazônicas. UTAM, Manaus, 29p. (monografia).

DENNIS, R. W. G. 1970. Fungus flora of Venezuela and adjacent countries. Kew Bull. additional ser. III. Royal Britanic Garden. 501p.

DIROL, D; FOURGEROUSSE, M. 1979. Schizophyllum commune Fr. In: Some wood destroying basidiomycetes. v. 1 . BOROKO, I.R. C. Wood Preservation. p. 129-139.
FIDALGO, O; FIDALGO, M. E. P. 1967. Dicionário micológico. Rickia, 2:1-232. supl.

GILBERTSON, R. L. 1980. Wood - rotting fungi of North America. Mycologia, $72: 1-41$.

GLBERTSON, R. L; RYVARDEN, L. 1987. North American polypores. Fungiflora, 2:437-885.

JESUS, M. A.; BONONI, V. L. 1991. Furgos em essências florestais da área da usina hidrelétrica de Balbina, Presidente Figueiredo, AM. Boletim ABPM, 70:19

KOTLABA, F; POUZAR, Z. 1964. Preliminary results on the staining of spores and other structures of homobasidiomycetes in cotton blue and its importance for taxonomy. Feddes Report, 69(2):131-142.

LOCQUIN, M. 1975. Guide des couleurs naturelles. I. De Taxia Fungorum.

MARTIN, G. W. 1934. Three new Heterobasidiomycetes. Mycologia, 26(3):261-265.

OFOSU-ASIEDU, A. 1979. Coriolopsis polyzona (Krs) Ryv. In: BOROKO, I. R. C. Some Wood destroying basidionycetes. v. 1, Wood Preservation; $39-$ 45 .

PINTO-LOPES, J. 1950. Poliporoses e fungos da decomposição da madeira em Portugal. C. Ciências Naturais. (2):53-108.

SINGER, R. 1975. The Agaricales in modern laxonomy. 3 ed., Vaduz, J. Cramer, $912 p$

TEIXEIRA, A. R. 1956. Métodos para estudo das hifas do carpóforo de fungos poliporáceos. São Paulo. Instituto de Botânica, 23p. 